NIST GCR 20-024

\title{
Open Standards for Flexible Discrete Manufacturing in the Model-Based Enterprise
}

\author{
Mark White \\ Eric Holterman \\ Tim Bakker \\ Larry Maggiano
}

This publication is available free of charge from:

https://doi.org/10.6028/NIST.GCR.20-024

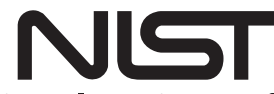

National Institute of Standards and Technology

U.S. Department of Commerce 
NIST GCR 20-024

\title{
Open Standards for Flexible Discrete Manufacturing in the Model-Based Enterprise
}

\author{
Prepared for \\ U.S. Department of Commerce \\ Engineering Laboratory - Systems Integration Division \\ National Institute of Standards and Technology \\ Gaithersburg, MD 20899 \\ By \\ Mark White \\ Eric Holterman \\ Tim Bakker \\ Larry Maggiano
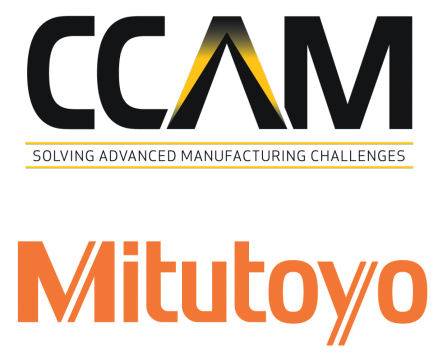

This publication is available free of charge from: https://doi.org/10.6028/NIST.GCR.20-024

September 2020

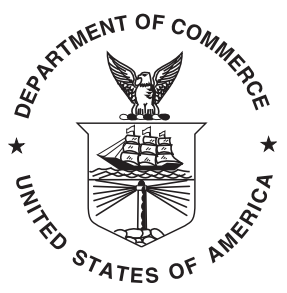

U.S. Department of Commerce Wilbur L. Ross, Jr., Secretary

National Institute of Standards and Technology Walter Copan, NIST Director and Undersecretary of Commerce for Standards and Technology 


\section{Disclaimer}

This publication was produced as part of cooperative agreement 70NANB19H083 with the National Institute of Standards and Technology. The contents of this publication do not

necessarily reflect the views or policies of the National Institute of Standards and Technology or the US Government. 


\begin{abstract}
The Commonwealth Center for Advanced Manufacturing is currently constructing an open, standards-based flexible manufacturing cell. The manufacturing cell must take in a product definition, machine the defined part, inspect it, rework as necessary, and provide a final dimensional report. In support of this effort, we define a flexible architecture based entirely on open standards. In this paper we look at standards which support a modelbased engineering approach to conveying product definitions to the manufacturing cell. The STEP AP242, QIF, and JT standards are compared and the various considerations for their use discussed. Finally we define a selected standard to use and the software that will be used in order to implement the chosen standard mix.
\end{abstract}

\title{
Key words
}

Flexible manufacturing; JT; MBD; MBE; Model based; PMI; QIF; Standards; STEP. 


\section{Who We Are}

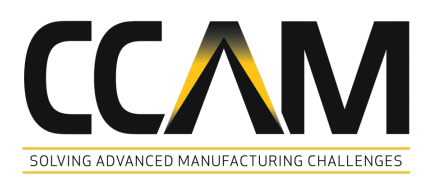

\section{Commonwealth Center for Advanced Manufacturing (CCAM)}

Founded in 2011 under a public-private partnership, the Commonwealth Center for Advanced Manufacturing (CCAM - pronounced: see-KAM) is an applied research center and not-for-profit 501c3 with membership from industry, university and government. This status and the structure of CCAM allows multiple universities and companies to collaborate in a research consortium, saving members valuable resources by accessing pooled talent, tools and technology to improve methodologies and increase product introductions.

\section{Mitutoyo}

\section{Mitutoyo America Corporation}

Founded in 1963, Mitutoyo America Corporation is part of, Mitutoyo Corporation, the world's largest global provider of measurement and inspection solutions offering the most complete selection of machines, sensors, systems and services. Mitutoyo's product lines encompass CMMs (coordinate measuring machines), vision, form and finish measuring machines, as well as precision tools and instruments, and metrology data management software. Mitutoyo America Corporation has a nationwide network of Metrology Centers and support operations that provide application, calibration, service, repair and educational programs to ensure that our 8,500+ metrology products will deliver trusted measuring instruments and solutions to our customers. 


\section{Table of Contents}

1 Introduction 1

2 Flexible Manufacturing Work Cell 1

2.1 Part flow 2

2.2 System architecture \& Standards Mapping 3

3 Standards of Interest 4

3.1 STEP AP242 (ISO 103030) 5

3.2 JT (ISO 14306)

3.3 ANSI/ISO QIF 3.0 8

3.4 MBE Standards of choice 11

4 Software Capability $\quad 11$

4.1 Design - Siemens NX 11

4.2 CAM Programming — Siemens NX 12

4.3 Metrology - Mitutoyo 12

$\begin{array}{lll}\text { 4.3.1 MiCAT Planner } & 12\end{array}$

4.3.2 MCOSMOS/GeoPAK/CAT $1000 \quad 13$

$\begin{array}{lll}\text { 4.3.3 MeasurLink } & 13\end{array}$

5 Conclusions 14

$\begin{array}{ll}\text { References } & 16\end{array}$

\section{List of Tables}

Table 1 MBE Standards $\quad 5$

\section{List of Figures}

Fig. 1 Flexible Manufacturing Cell 2

Fig. 2 Sample Part Workflow 3

Fig. 3 Architecture and Standards for the Sample Flexible Manufacturing Cell 4

Fig. 4 AP 242 ed2 Capabilities 6

Fig. 5 Annotated JT File $\quad 7$

Fig. 6 QIF Soccer Ball 9

Fig. 7 Check-Mate Function in NX 12

Fig. 8 MiCAT Planner 13

Fig. 9 MeasurLink Modules $\quad 14$ 


\section{Glossary}

BOM - Bill of Material

CCAM - Commonwealth Center for Advanced Manufacturing

GD\&T - Geometric Dimensioning and Tolerancing as defined by the ASME Y14.5 standard. This is the ASME version of GPS.

GPS - Geometric Product Specifications as defined by the ISO 1101 standard. This is the ISO version of GD\&T.

LOTAR - Long Term Archiving and Retrieval

MBE - Model Based Engineering

PMI - Product Manufacturing Information 


\section{Introduction}

Industry continues to move towards a model based digital future where open standards facilitate the integration of product, production, and business data. Standards such as STEP AP242, JT, QIF, and MTConnect are seen as fundamental enabling technologies for smart manufacturing and are key differentiators for near term business success. By standardizing data structures and semantics for the digital thread connecting products, processes, and resources, these technologies enable improved efficiency, flexibility, and process quality control. Challenges to standardized digital definitions still exist; however, these smart manufacturing standards continue to be actively developed, expanded, and harmonized where possible through the efforts of NIST, ISO, AMT, DMSC, and many others in both the public and private spheres.

Open standards allow for the creation of a robust, extensible digital backbone for the modern factory. They hold incredible value to manufacturers interested in being able to select the right equipment and configuration for their job rather than being locked into a single vendor ecosystem or having to continually support a web of custom equipment interfaces. The ability to easily add and remove equipment provides businesses the ability to select the optimal piece of equipment for their needs, thereby achieving a higher ROI and greater flexibility for pursuing additional work.

Pairing open standards together with model based engineering provide OEMs and suppliers a consistent language by which to communicate model based product definitions. It also allows for more efficient workflows since both parties may purchase and use software that is right sized for their business. Pairing this with an open method of conveying metrology data back to OEMs provides a sudden wealth of information for process control, reasonable tolerances, etc. that may be used to improve future designs, time to manufacture, and cost resulting in a win-win for the supplier and the OEM.

The benefits of an open standards based digital backbone for discrete manufacturing are many and varied. The following sections discuss the physical/digital test environment constructed at CCAM and attempt to shed light on some of the many standards that are available within the model based engineering domain. A separate CCAM whitepaper covering the network communication \& semantics will be released shortly.

\section{Flexible Manufacturing Work Cell}

CCAM's sample flexible manufacturing work cell is a self-contained, extensible, manufacturing cell with a dedicated digital infrastructure enabling data collection and exchange and device control. It includes a heterogeneous set of equipment that can perform a set of individual process steps and be automatically adapted to variations in part designs, process flows, etc. The cell shown in Figure 1 is composed of a Mitutoyo MiSTAR 555 shop floor CMM, Hurco VM10i CNC milling machine, collaborative robot \& integrated material handling solution by Simplimatic, and Cisco's IOx-enabled industrial ethernet network \& edge 
compute hardware. Other equipment combinations may be quickly implemented by leveraging the open standards mix and existing digital backbone of the cell.

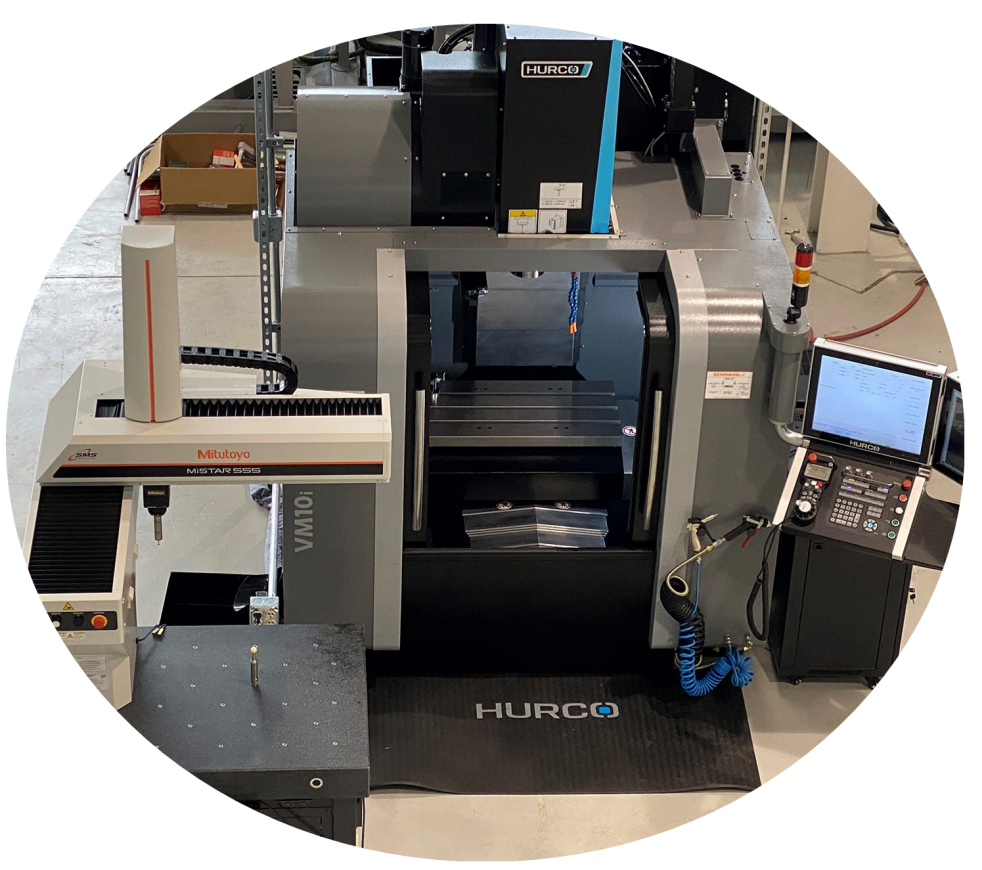

Fig. 1. Flexible Manufacturing Cell. The cell is being constructed on CCAMs manufacturing floor but may easily be replicated to new locations and with different equipment mixes.

\subsection{Part flow}

Figure 2 below depicts one use case in which a part is introduced to the work cell, machined at the $\mathrm{CNC}$, inspected by the CMM, and then either passed to the next production step, re-worked, or scrapped. As discussed previously, open standards are the key to achieving an attractive ROI for this level of digitalization. If a manufacturer must reinvest in software for every permutation of part or process then it becomes hard to justify the investment (ergo the relatively slow pace of digitalization in non-commodity applications). Conversely, open standards allow a manufacturer to invest one time in a cell architecture and then expand over time to satisfy business needs. 


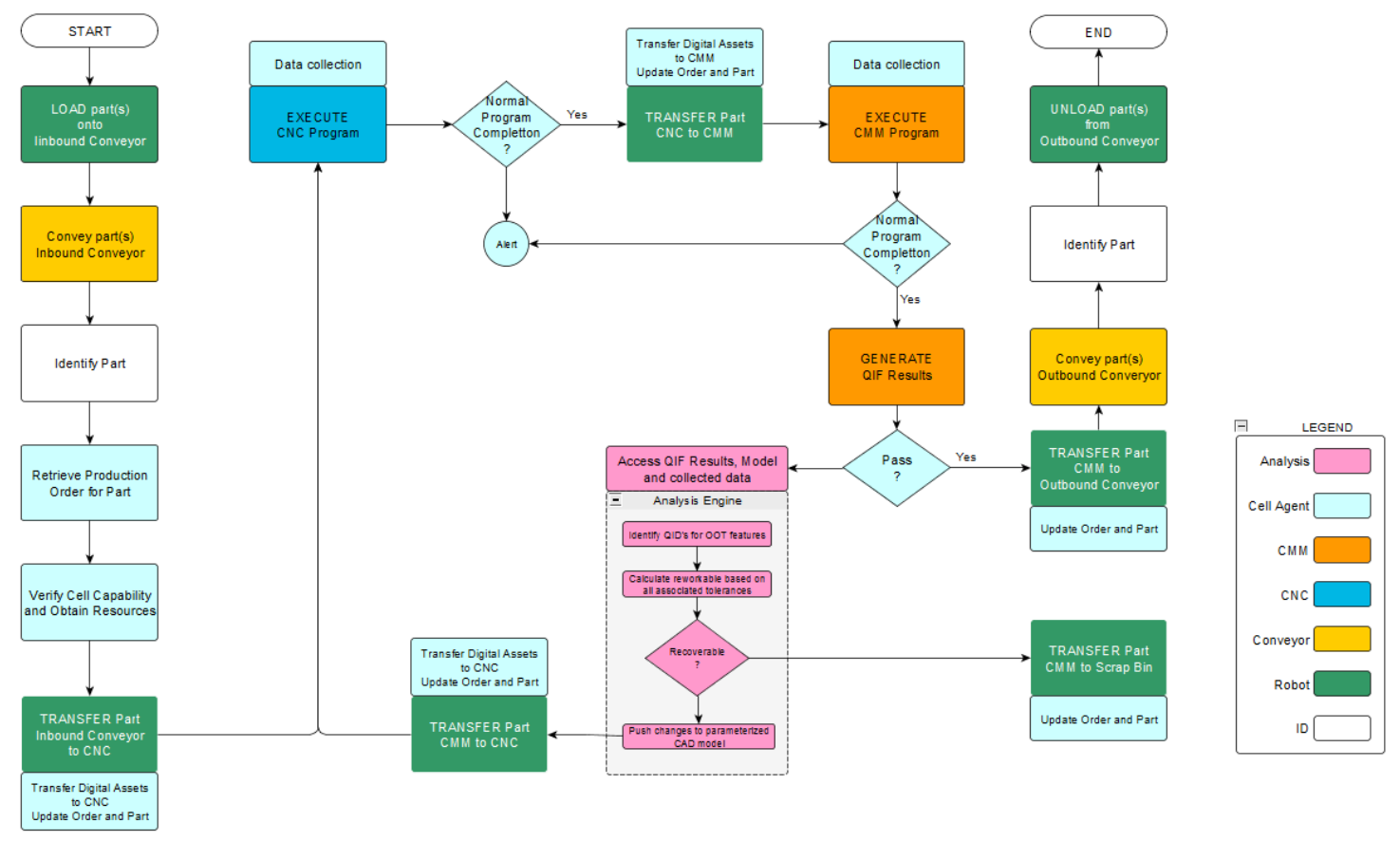

Fig. 2. Sample part workflow. This workflow represents full automation from part entry until part disposition including automatically defining if rework is needed and how to complete that rework.

\subsection{System architecture \& Standards Mapping}

Figure 3 depicts the system architecture and standards selection for the flexible manufacturing cell. Within this architecture, the most critical standards for our implementation are STEP AP242, QIF, and MTConnect. These allow for clearly understood messages between devices along with clarity of part definition and manufacturing results. MTConnect allows for stateful representations for all entities, which are processed by micro-services (within the supervisory control services block) to enable decisions based on the product definition (AP242) and results (QIF Results) or resources scheduling (QIF Resources). For equipment without a native MTConnect agent, such as the robot used for material movement, we demonstrate how to bring them in through a device service that acts as a translation layer thereby allowing for a consistent standards based approach for all cell entities. This architecture and the related open standards for communication and semantics are the subject of a separate CCAM whitepaper in on flexible discrete manufacturing. 


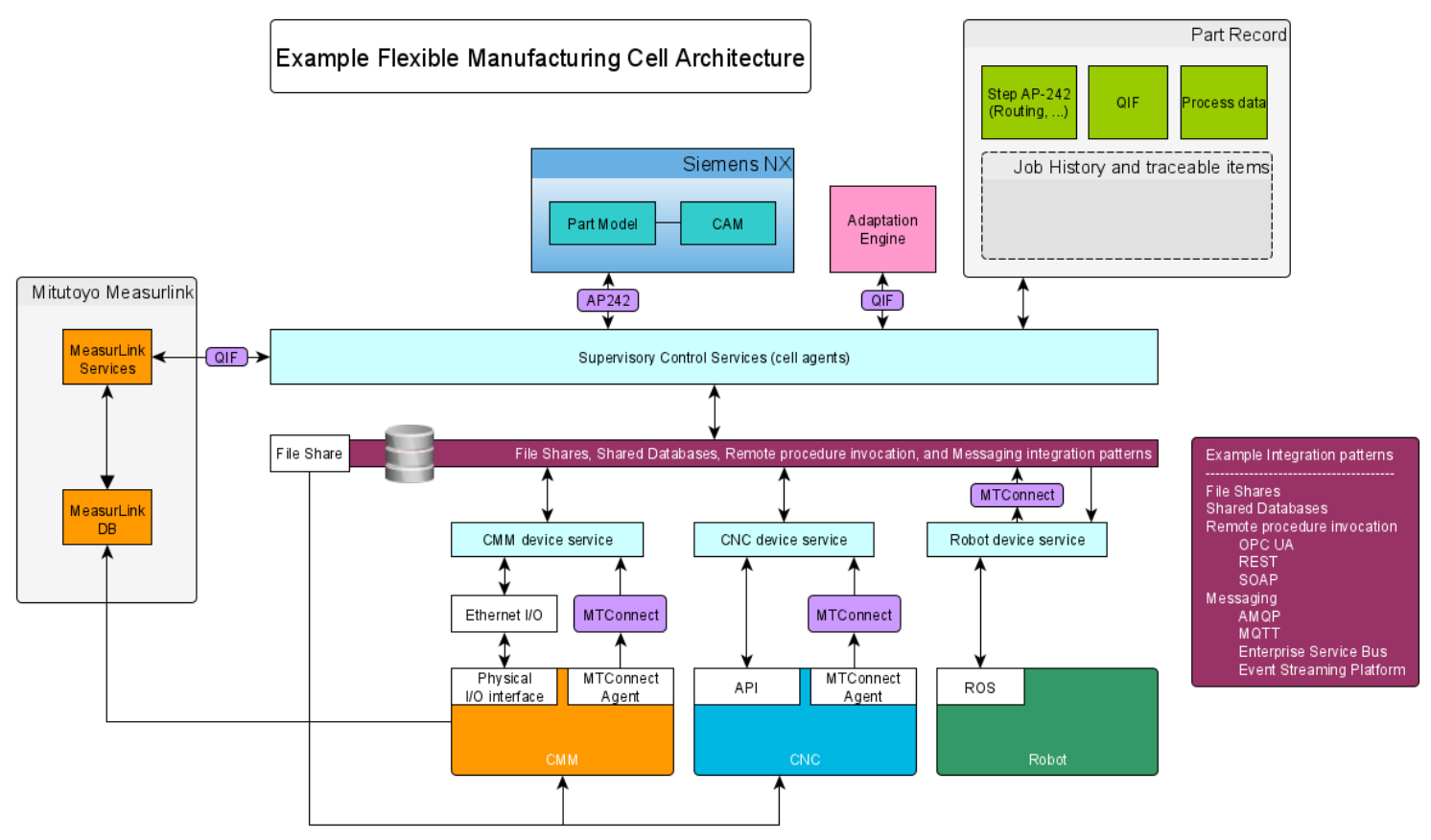

Fig. 3. Architecture and Standards for the sample Flexible Manufacturing Cell. The primary standards used for sharing data within the cell are QIF, AP242, and MTConnect.

\section{Standards of Interest}

Model based engineering (MBE) is, "an approach to product development, manufacturing, and lifecycle support that uses a digital model to drive all engineering activities" [1]. By having OEMs and their supply chains be model based enterprises (practicing MBE) the processes of quoting, manufacturing, inspecting, and reporting can be heavily streamlined and error prone manual steps can be substantially reduced or eliminated. This results directly in shorter turnaround time, reduced quality costs from misinterpreted requirements, and therefore reduced costs to manufacture goods helping both OEMs and suppliers improve profits.

The advantages of open standards based MBE for OEMs and their supply chains are two sides of the same coin. For the OEMs a single data packet can be used for all suppliers with no lost time as agreeable data formats are aligned on. They can also ensure that their quality standards are met, process traceability is in place, and that required information is reported back and easily digested. This helps to reduce the costs associated with helping suppliers troubleshoot challenges. At the same time, open standards based job packets coming from OEMs help suppliers reduce their own costs. Shops receiving orders from multiple customers no longer need to purchase multiple proprietary, and frequently expensive, CAx solutions in order to support their different customers. They can purchase the right sized solution for their business and focus on building technical depth in the single package. Downstream manufacturing and inspection steps can then be generated directly 
from the model which, in the absence of a drawing, becomes the master product definition. In addition, some standards such as QIF 3.0, provide the ability for an OEM to clearly define inspection rules to ensure inspection needs are met, along with a consistent framework for sharing data from the supplier back to the OEM.

By way of contrast, proprietary MBE solutions are quite widespread and can offer many of the same technical capabilities. However, closed solutions pose numerous risks to adopters. Namely, once an organization has fully invested in a single proprietary ecosystem, their supply base is not diverse and price negotiations can be challenging. In addition, when working with outside firms (OEM, supplier, contractors) a translation step must take place or the other party must purchase the same CAx package in order to collaborate. This adds cost to the manufacturing lifecycle which may or may not be countered by the efficiency of the single vendor ecosystem. Where multiple proprietary solutions are encountered, such as between quality and design departments, handoffs require manual recreation of work or custom translators.

Table 1. MBE Standards

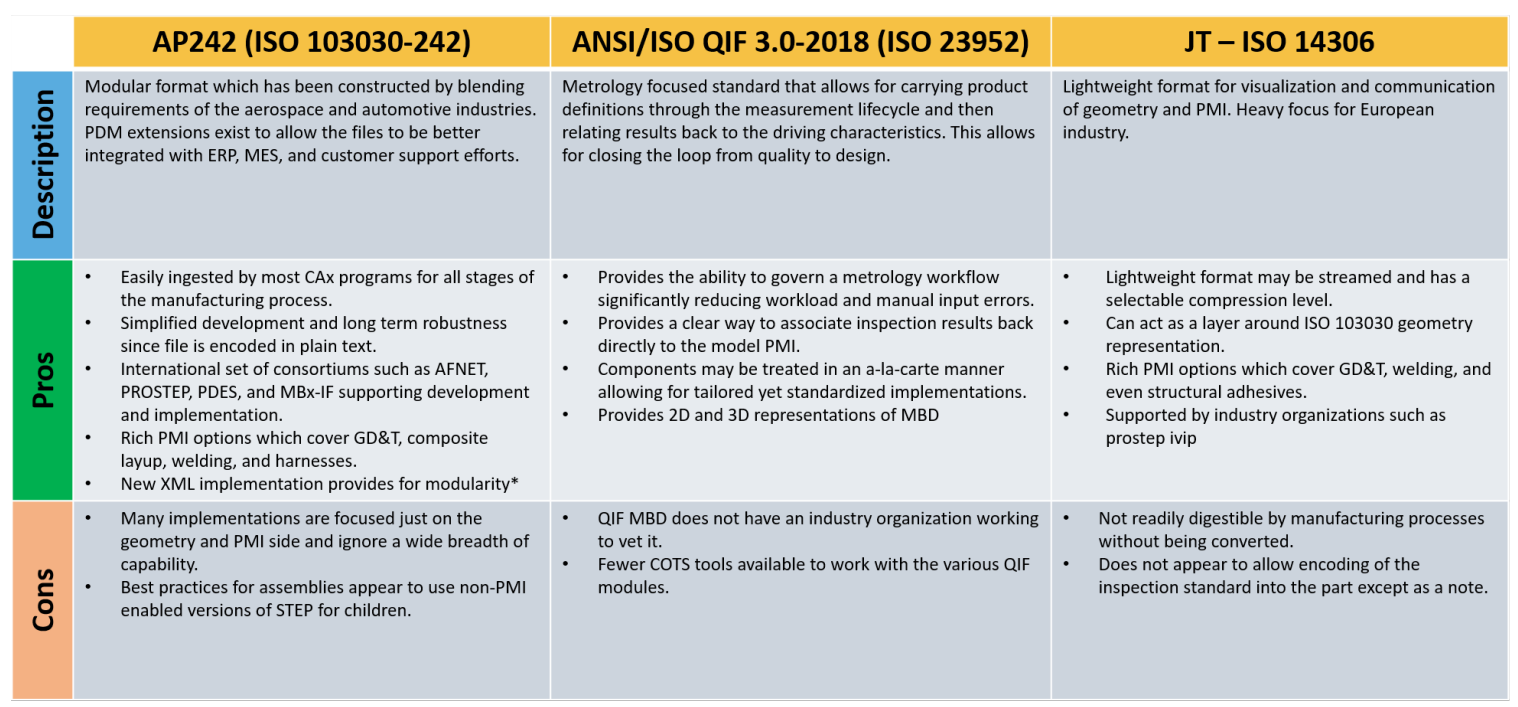

In looking at open standards to leverage in an MBE solution, we landed on three separate standards which are discussed below; ISO 103030 part 242, also known as STEP AP242, ISO 14306 JT, and ANSI/ISO QIF 3.0. A high level overview may be seen in Table 1: MBE Standards.

\subsection{STEP AP242 (ISO 103030)}

STEP AP242 is a modular format focused on "product data representation to support $\mathrm{CAD} / \mathrm{CAE}$ and PDM data exchange, system integration, visualization, and long term 
preservation of product information" [2]. AP242 was constructed by blending and improving on the formats built for aerospace (AP203) and automotive (AP214) industries. It extends these prior formats with the inclusion of PMI, extensions for PDM \& MES, supporting kinematic models, and many other improvements (Figure 4). The AP242 module was further extended by ISO 10303-3001 which introduced a business object model defined by XML rather than EXPRESS schemas.
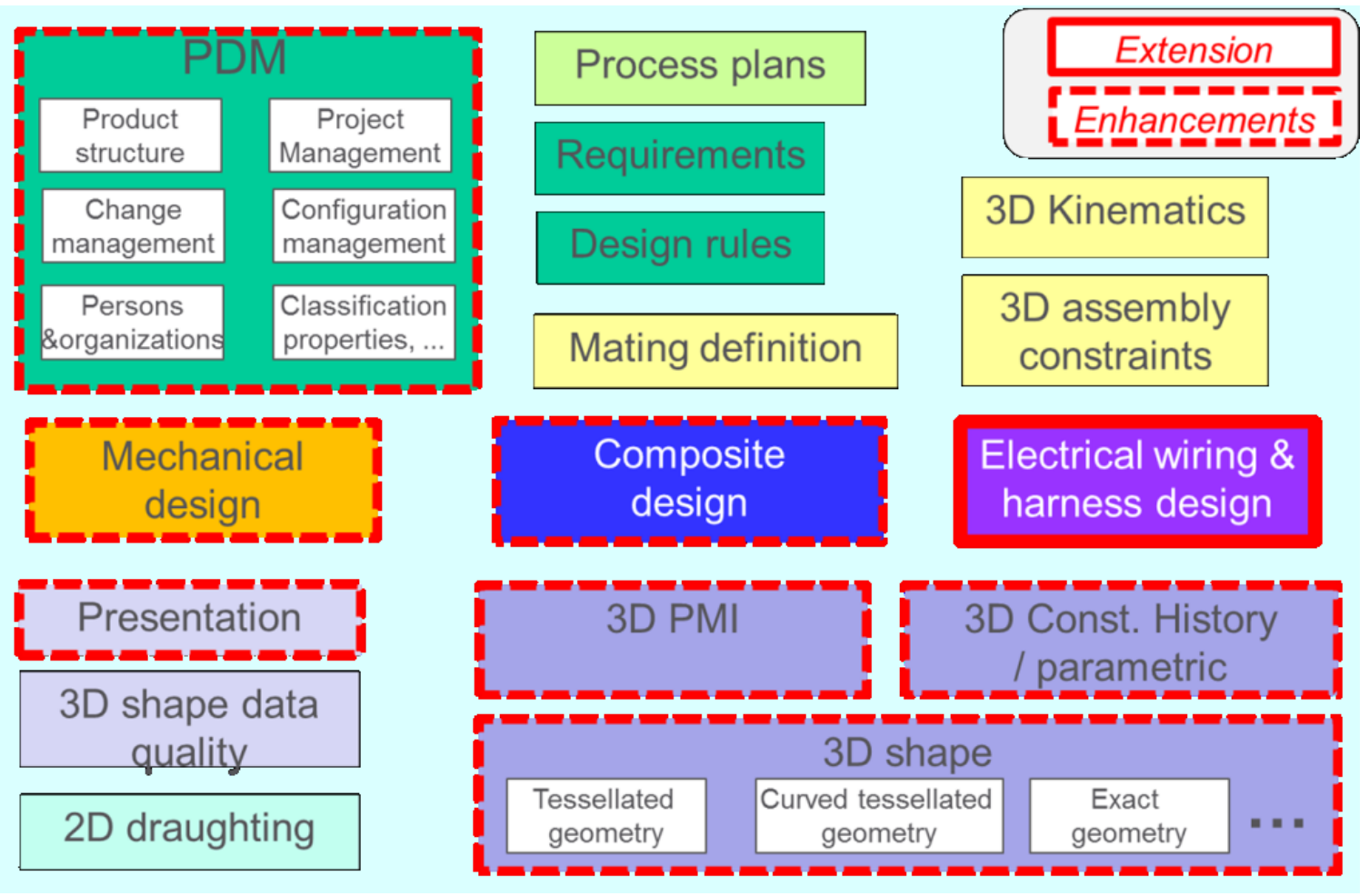

Fig. 4. AP 242 ed2 Capabilities. [3]

When looking at STEP as a file format we find a number of substantial advantages. Key among them are the broad support for digesting STEP among CAx packages, the plaintext encoding which directly supports long term archiving and retrieval (LOTAR), rich PMI capabilities, and with part 3001 the ability to create assemblies via external references. In addition the STEP standards are supported internationally by industry through consortiums such as AFNet, prostep ivip, PDES, Inc., and the MBx Interoperability Forum (MBx-IF). All of these contribute to a standard that could be regarded as a one stop shop for MBE requirements within an open standards framework.

Benefits aside, there are also some disadvantages to STEP files. As a notable contrast to ISO 14306 (JT) STEP files do not carry rich visualization data. In addition they tend to be a larger file size than even uncompressed JT files. When using the external references model, current CAX-IF best practices recommend that the final geometry model is either AP203 or AP214 which means that multiple documents would have to be transmitted in 
order to carry semantic PMI [4]. Upcoming but as-yet non finalized revisions to these recommendations will start permitting AP242 for external references (also termed leaf nodes).

\subsection{JT (ISO 14306)}

Jupiter Tessellation (JT) files are a lightweight format for visualization and communication of geometry and PMI. This file type has already been widely adopted by the automotive industry and is a particular focus among European industry [5]. The industry organization prostep ivip has been key in driving adoption and harmonization of JT. The JT format was transitioned from being Siemens proprietary over to ISO in 2011. Following the standards release a JT Industrial Application Package was specified by prostep ivip as DIN Spec 91383 which allowed for neutral and royalty-free implementation of JT with a more rapid update cycle than that provided by ISO [6].

The JT format advantages are perhaps best captured by its rich visualization capabilities and reduced file size. For the model based enterprise it's important to be able to conduct digital mock ups (DMUs) where very large models, frequently entire vehicle BOMs, are loaded. Visualization support allows for transmitting of colors, transparency, lighting, etc. data forward to other departments such as ergonomics and marketing. At the same time support for lossless or a fine-tuned lossy compression allows for rapid transferal of the data at just the right level of detail for the downstream user.

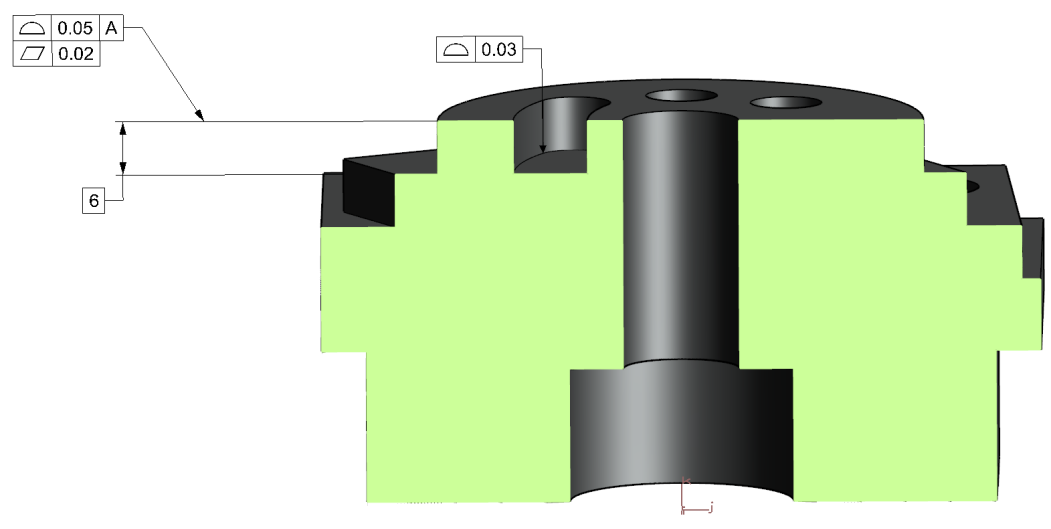

Fig. 5. Annotated JT file. The cross section is carried forward in JT as a model view.

The JT file format supports a rich set of PMI options such as GD\&T, weld callouts, and even structural adhesives. However, at the current time there does not appear to be a dramatic difference in PMI carrying capability between STEP AP242 and JT. There are however differences in which elements are supported (i.e. JT has structural adhesive whereas STEP has electrical harnesses). One point of interest for downstream inspection use is that the JT file format has support for a PMI measurement point entity (ISO 14306-2017: 9.2.1.9). This entity can store measurement point locations and normal vectors thereby 
conveying to a CMM package exactly where to measure. At the time of writing it appeared that only NX CMM had the capability to work with these collections in that manner, but that functionality has not yet been confirmed by the authors.

The JT format does have a couple of drawbacks. The largest is that it does not currently enjoy broad support among CAM systems. For direct use of off the shelf software, STEP seems to be better supported. The PMI options appear to be quite comprehensive but annotating which standard (ASME Y14.5 or ISO 1101) is done through general notes rather than a pre-set Metadata tag per the 2017 standard. With edition 3, the ISO standard will catch up to Siemens JT v10.5 and include a callout for embedding the inspection standard. The result of these two issues is that additional processing is required. For the first, a model conversion and quality check step needs to be implemented twice. First the model is converted from the design master to JT and then again from JT to STEP. In a supplier scenario the JT would be compared against the proprietary format design master then sent to the supplier. The supplier would be responsible to convert over to STEP and compare back to the JT. This means that in the absence of direct COTS support for importing JT files, we end up using STEP anyways. This should change as more manufacturing equipment and software suppliers begin to support the JT format. For the second issue, the inspection standard to use will need to be transmitted independently or somehow scraped from the note PMI until edition 3 is implemented. Both of these open the door to mistakes.

It is important to note that the automotive industry is currently looking very heavily at the STEP AP242 XML business object model for PLM, metadata, kinematics, etc. combined with JT for conveying geometry, visualization, and PMI [7]. This solution is very attractive as it maintains product structure and additional product information while still providing a very lightweight file format. It is an ideal blending of the two standards but still does not resolve the issue with the current lack of COTS support for JT files in manufacturing. From an open standards perspective another challenge is that many consumers of the JT format are actually consuming the, very capable but proprietary, Siemens format. The open standard follows along with the proprietary but has a time lag for new features being implemented.

\subsection{ANSI/ISO QIF 3.0}

ANSI/ISO QIF (ISO 23952:2020) is a metrology focused standard that allows for carrying product definitions through the measurement lifecycle and relating results back to the driving characteristics. This allows for closing the loop from quality to design. It also is defined using XML files in a modular manner from which elements may be picked à la carte. This modular nature means that businesses can implement a standards based solution that is right sized for them and coexists with other standards they may choose. For example, a manufacturer may simply want to report results and statistics to a customer. In this case they would only implement QIF Results and Statistics, leaving their CAD choice open and retaining their existing metrology planning/inspection workflow. The standard itself is developed and maintained by the Dimensional Metrology Standards Consortium (DMSC) 
and was first accepted as an ANSI standard in 2013.

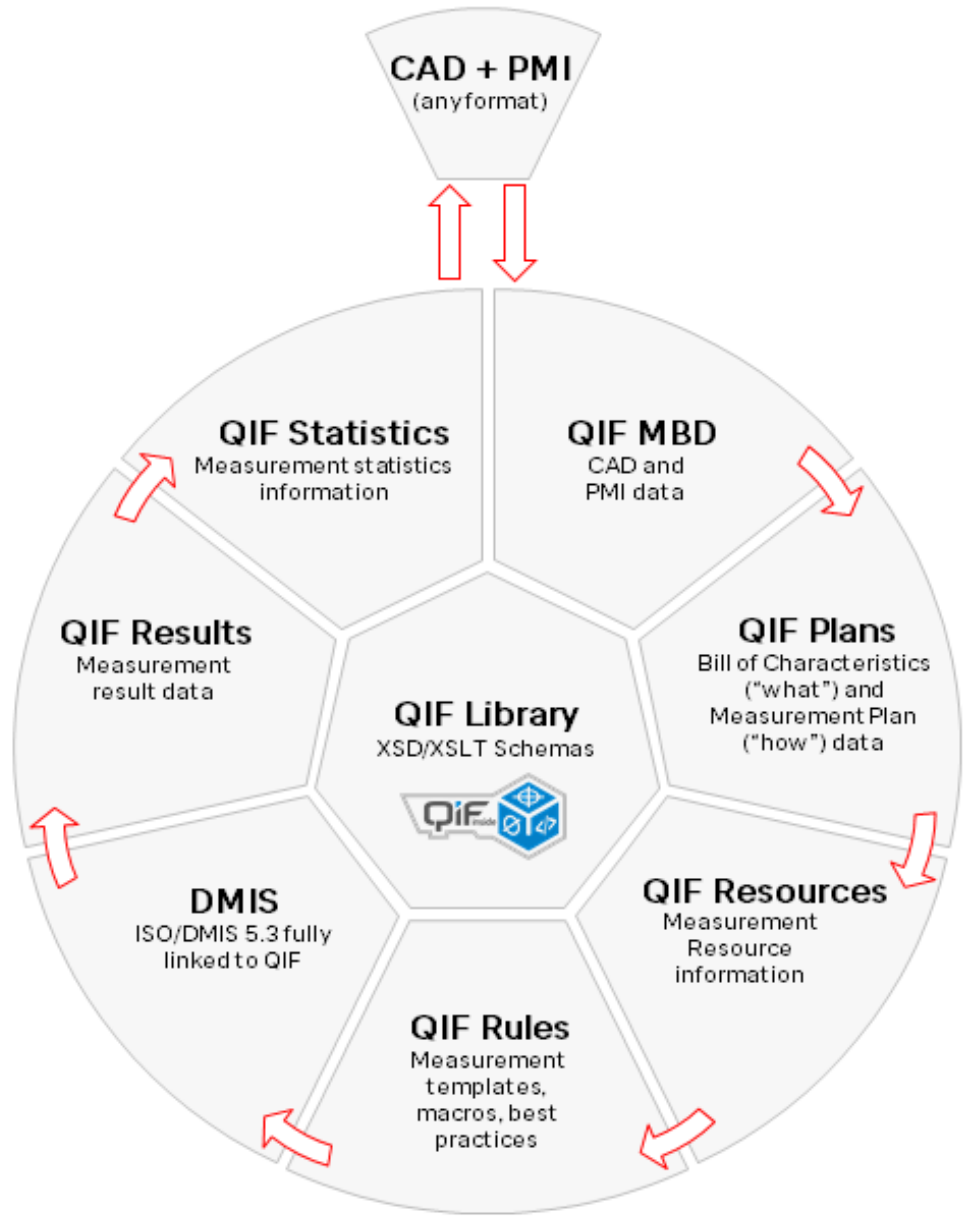

Fig. 6. QIF Soccer ball. Six segments are supported. Execution, here shown as DMIS, is not a module of QIF. [8]

With the exception of QIF MBD, which is a metrology focused geometry corollary to AP242 and JT, QIF is uniquely designed to meet the needs of metrology users. Each module is constructed to permit automation or streamlined execution in the metrology workflow. QIF plans for example break out all features and characteristics into a bill of characteristics, this replaces the traditional requirement of creating a bubble drawing to link inspection results back to drawings. Since each feature and characteristic in the QIF MBD file is referenced with a universally unique identifier (UUID), the results can be tagged directly back to the model callouts without the additional marked up drawing. QIF Resources may be maintained at the factory level as a library of all inspection resources available so that they can be directly associated to an inspection task. QIF rules define the methods by which fea- 
tures and characteristics are measured. This permits for rapid development of inspection programs since best practices or customer requirements may be captured as a template. By applying the template and resource to the plan, an inspection program may be generated. The metrologists role then becomes one of applying metrology knowledge to tweak the generated programs and maintaining the resources and rules which are used to generate programs in the first place! In one case study, a 30\% reduction in CMM programming time was realized through the use of QIF with the researchers recognizing a clear potential for up to $70 \%$ time reduction [9].

Continuing around the QIF soccer ball (Figure 6) comes to an inspection program defined in DMIS. As of QIF 3.0, "the DMIS application, ... is a placeholder for a future QIF execution model that is not a part of QIF." Therefore whichever application used to ingest the QIF rules will need to independently be able to understand them and generate a part program. QIF Results are what traditionally get placed into an inspection report. However, using QIF they are all tied back to the UUID of the feature and characteristic of the MBD annotated model. This opens avenues for analyzing measurement data results for rework needs and passing back the part and clear instructions for change to prior manufacturing steps. QIF Results are then analyzed to form QIF Statistics, a repository for statistical process control (SPC) data on a part allowing to see and react to short and long term trends in the manufacturing process.

QIF MBD is the QIF standards take on conveying geometry similar to AP242 and JT. This has led to harmonization efforts between the AP242 and QIF standards to try and address overlap. QIF MBD covers much of the same territory as AP242 with regards to geometry and PMI. QIF MBD geometry however is wrapped by metrology features (i.e. cylinder, plane, parallel planes, etc.) which are in turn referenced by characteristics such as GD\&T feature control frames [10]. However, beyond just the overlap with AP242, there are some additional drawbacks to QIF MBD. The most notable one is the lack of broad support. Currently there are no industry consortiums such as MBx-IF for AP242 which are working to validate and test QIF MBD implementations; however, NIST has created a conformance validation tool similar to the STEP AP242 analyzer. This may be in no small part due to the low number of commercial translators which can work with QIF MBD files natively. QIF MBD as a way of communicating product definitions between businesses also faces the same struggles as JT files. Namely the lack of native support for the format among manufacturing software packages. This means that a model conversion to a more generally supported format will be required. So the QIF MBD must be verified against the master and then the supplier is forced to convert and verify against the QIF MBD file.

Similarly, since it is focused on metrology, support for kinematics and PLM type data is lacking. However, remembering that the QIF standard may be taken à la carte; all that is necessary to leverage the benefits of STEP, JT, or any other format is the ability to place a UUID anchor for different elements within the file (QIF 3.0, 7.5.4.4). In this way the advantages of QIF for streamlining and mitigating error in the quality workflow may be maximized while the PMI/geometry representation standard may be chosen as whichever is the best organizational fit. 


\subsection{MBE Standards of choice}

CCAM currently recommends the use of STEP AP242 for communicating product definition and PMI. This is also the geometry representation that will be used for manufacturing CNC paths. Metrology will be handled through an à la carte implementation of QIF leveraging QIF plans, resources, rules, results, and statistics.

In the future leveraging AP242 XML with JT individual parts may be a very powerful method by which a consistent approach may be used for conducting DMUs, collaborating with offsite contractors, and providing manufacturing data. Where LOTAR requirements need to be met, STEP AP242 models may be converted, compared for accuracy, and stored based on the proprietary base format. The key cornerstone for transitioning to JT will be greater support among CAx software for the format. Once the core systems required for a given manufacturing process have JT support, our recommendation is to re-consider AP242 XML with JT while still using QIF for the metrology workflow. A significant caveat is that translators must support the ISO 14306 version of JT rather than only for the Siemens proprietary version of JT in order to achieve the open standards objective.

\section{Software Capability}

\subsection{Design - Siemens NX}

Almost any 3D CAD package may be used for creation of the design master provided that it supports PMI annotation and export to STEP AP242. Here at CCAM we will be using Siemens NX to create our master authority parts. In addition to the features just mentioned, it also can perform model comparisons, verify the quality of PMI to GD\&T and GPS standards, and export JT files. This makes it an effective choice not just for immediate deployment but also testing multiple different blends of standards within an automated cell. 


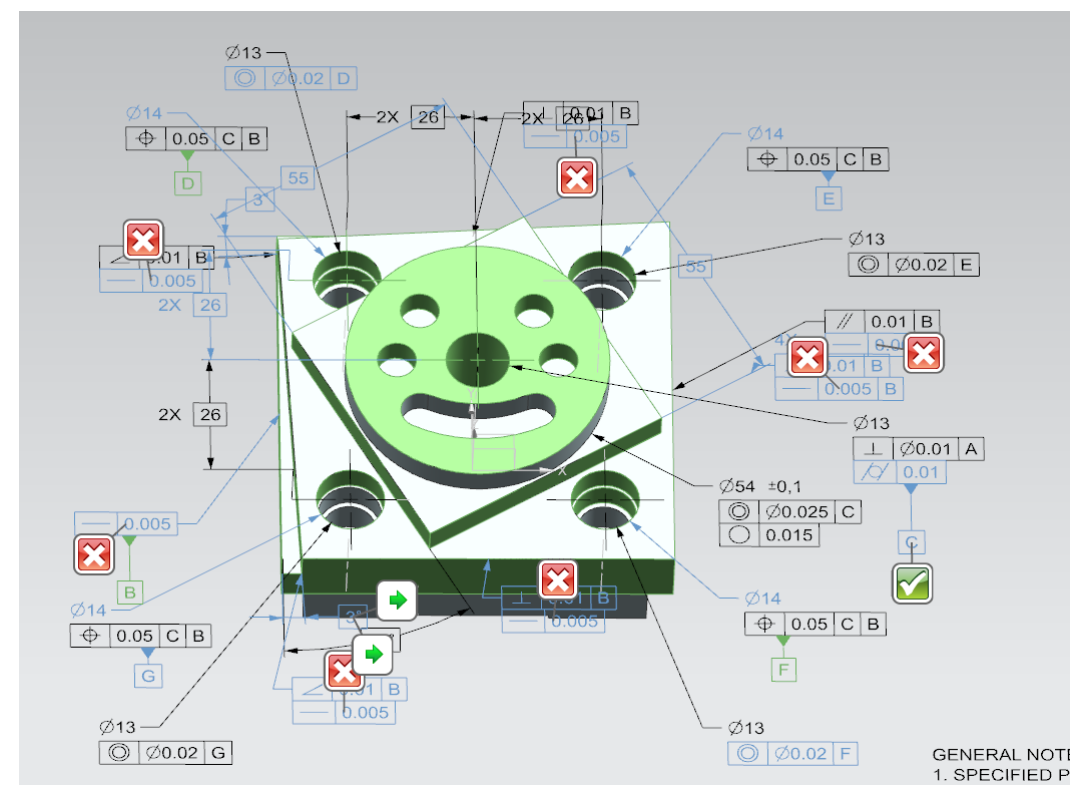

Fig. 7. Check-Mate function in NX. This allows validating PMI callouts against ISO 1101 and ASME Y14.5.

\subsection{CAM Programming - Siemens NX}

The Siemens NX CAM environment is ideal for authoring CAM programs within the automated cell. It offers support for both JT and STEP AP242 part documents and can post-process to multiple different machining centers. Within the same environment, the machining center may be simulated thereby giving the CAM programmer confidence that there will be no crashes and an opportunity to optimize paths as they see fit.

\subsection{Metrology - Mitutoyo}

\subsubsection{MiCAT Planner}

MiCAT Planner is an innovative software package developed by Mitutoyo for analyzing annotated $\mathrm{CAD}$ models and generating inspection programs based on rule sets and user settings. Once the CMM of interest is configured within the software, the metrologist loads the part model and any supported semantic PMI is parsed to create features and inspected characteristics. Rule definitions may be modified within each project to then implement best practices or customer requirements. These rule definitions are extremely close to QIF rules.

Once the program is generated it may be simulated virtually to ensure no collisions and that the metrologist is comfortable with how it has decided to run the inspection. Afterwards it may be uploaded to Mitutoyo's MCOSMOS to run on the real machine and inspect components. 


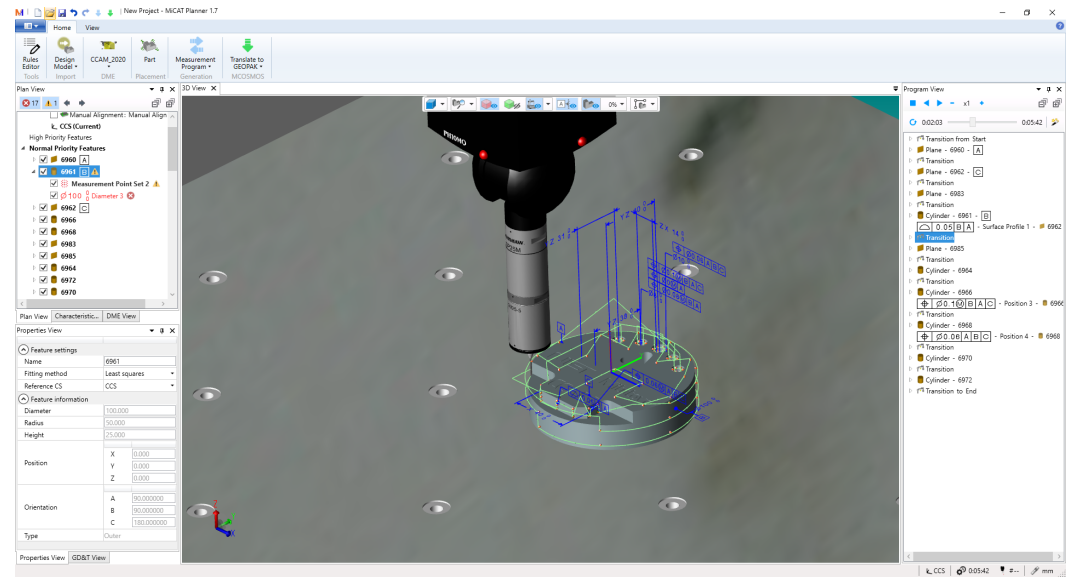

Fig. 8. MiCAT Planner. Part with PMI is loaded and then a program run on a virtual CMM.

MiCAT Planner currently supports importing of numerous proprietary formats as well as STEP AP203, AP214, and AP 242.

\subsubsection{MCOSMOS/GeoPAK/CAT 1000}

The MCOSMOS, GeoPAK, and CAT 1000 software packages from Mitutoyo will be used to execute part inspection. These packages are used to select the part program to run, make any detailed changes to the automatically generated program from MiCAT Planner, execute the inspection, and export results to Measurlink.

\subsubsection{MeasurLink}

Mitutoyo MeasurLink is a rich data management and SPC software. It consists of a six different modules and a centralized database in order to offer a comprehensive quality management system (QMS). For the purposes of an automated cell, rather than overall factory management, MeasurLink will be used for collection of inspection results, compilation of SPC data, and exporting QIF Results and QIF Statistics data for ingestion elsewhere. 


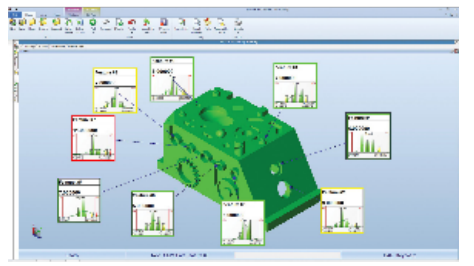

(a) Real-Time Professional 3D

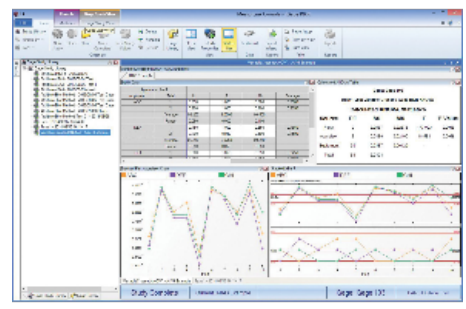

(d) Gage R\&R

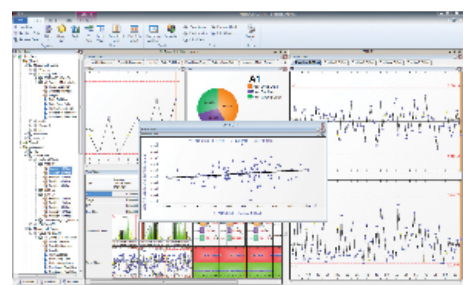

(b) Process Analyzer Professional

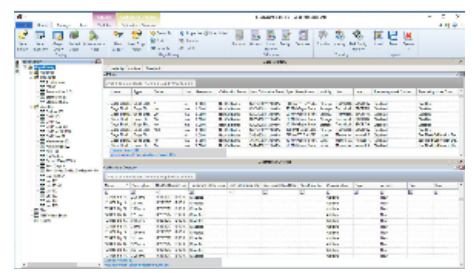

(e) Gage Management

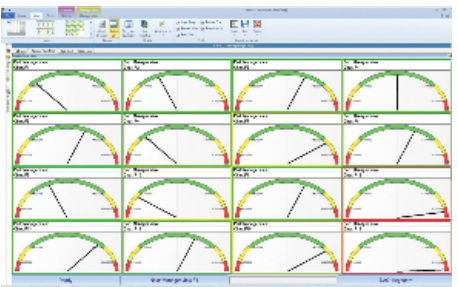

(c) Process Manager

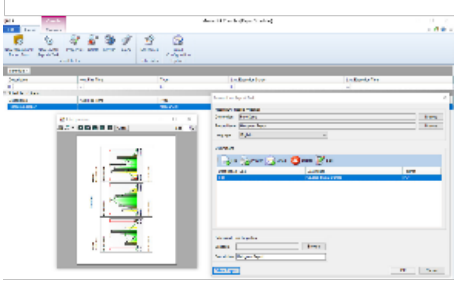

(f) Report Scheduler

Fig. 9. MeasurLink modules. Six modules make up the MeasurLink suite and can manage everything from results, to SPC, to gauge calibration schedules. Within the automated cell we will focus on results collection and statistics reporting as QIF files.

\section{Conclusions}

Today's manufacturers are under constant pressure to perform more work with fewer resources, lower costs, and reduced lead times. In the past this has been achieved through greater levels of automation and frequently satisfied via custom solutions targeted at specific applications. In order to continue increasing automation, solutions need to become more flexible to permit manufacturers to satisfy tighter product development cycles or customer lead time requirements. Selecting open standards as the foundation of these future flexible discrete manufacturing cells is crucial in enabling greater agility and a shift away from proprietary or custom solutions.

The ability to clearly define and communicate product definitions in a computer readable manner is crucial to flexibility in modern manufacturing. Model based engineering, and by extension the model based enterprise, is a strong answer to this need. For OEMs and suppliers, the AP242 standard (ISO 103030-242) provides a plain-text representation of geometry and semantic product manufacturing information that is readable by almost all CAD/CAM software packages. Pairing this solution with the ANSI/ISO QIF standard provides for tying metrology planning and reporting directly back to the part model, reducing programming times and allowing a consistent OEM-supplier quality language for communicating metrology results.

In collaboration with NIST, CCAM is currently constructing a demonstration cell for 
flexible discrete manufacturing that showcases one implementation of the concepts discussed above and in a soon to be released networking/communication standards whitepaper. Within this cell we are leveraging Mitutoyo's hardened shop floor CMM, the MiSTAR 555, a Hurco VM10i CNC mill, an integrated UR5 \& MiR 200 material handling solution by Simplimatic, and Cisco's IOx-enabled industrial ethernet network \& edge compute hardware. This cell will provide a rich environment for the development and refinement of an open standards based cell and is slated to be fully operational by the end of 2020 . 


\section{References}

[1] Lubell J, Chen K, Frechette S, Horst J, PHuang (2012) Model based enterprise / technical data package summit report (NIST), NIST Technical Note 1753.

[2] Project SA (2020) Ap242 - why the convergance, . Available at http://www.ap242. org/why-ap242.

[3] (2014) Development of step ap242 ed2 "managed model based 3d engineering".

[4] Boy J, Rosche P, Trautheim A (2020) Recommended Practices for External References with References to the PDM Schema Usage Guide Release 2.1, .

[5] IVIP P (2020) Jt workflow forum. Available at https://www.prostep.org/en/projects/ jt-workflow-forum/.

[6] IVIP P (2020) Jjt standardization. Available at https://www.prostep.org/en/projects/ jt-standardization/.

[7] Katzenbach A, Handschuh S, Vettermann S (2013) 6th Programming Languages for Manufacturing (PROLAMAT), .

[8] Digital Metrology Standards Consortium ID (2018) Quality information framework (qif) - an integrated model for manufacturing quality information (Digital Metrology Standards Consortium, Inc. (DMSC)),

[9] Herron J, Andujar L, Gelotte R (2019) A qif case study - maintaining the digital thread from oem to supplier, . Available at https://www.action-engineering.com/wp-content/ uploads/14-Paper-Herron-Gelotte-OEM-to-Supplier-Handoff-v06.pdf.

[10] Brown C (2020) Qif 101: Understanding qif basics, . Available at https://qifstandards. org/resources/. 